\title{
Predictors for Retrograde Ureteral Stenting Failure as an Initial Drainage Method for Emergent Complicated Acute Calculus Obstructive Uropathy
}

\author{
M. A. Elbaset, $₫$ Mohamed Edwan, Rasha T. Abouelkheir, Rawdy Ashour, Mohamed Ramez, \\ Abdalla Abdelhamid, Yasser Osman \\ Urology and Nephrology Center, Mansoura University, Egypt
}

\section{Abstract}

Objective To define predictors for initial retrograde ureteral stenting (RUS) failure with the need for the percutaneous nephrostomy (PCN) insertion as a drainage method in patients with complicated acute calculus obstructive uropathy.

Methods We undertook a retrospective evaluation of patients who presented with complicated obstructive calculus uropathy (acute renal failure or obstructive pyelonephritis) between January 2016 and January 2020. Patients in whom there was failure to visualize ipsilateral ureteric orifice and those with extrinsic ureteral obstruction were excluded. Patient demographics and radiological data including stone site, hydronephrosis grade, maximum transverse stone diameter, periureteral density (PUD) and pericalcular ureteric thickness (P-CUT) at the maximum transverse stone diameter were assessed using non-contrast computed tomography at the time of admission.

Results The study included 256 patients who were managed initially by RUS trial. Of them, 48 (18.8 \%) had RUS failure. The presence of acute pyelonephritis, increased maximum transverse stone diameter $\geq 9.5 \mathrm{~mm}, \mathrm{P}$-CUT $\geq$ $7.5 \mathrm{~mm}$, and PUD at stone level $\geq 17.5 \mathrm{HU}$ were risk factors associated with RUS failure $(P=0.007,0.002,<0.001$, and $<0.001$, respectively).

Conclusion Initial radiological stone and ureteric characteristics, in addition to the clinical diagnosis of obstructive pyelonephritis, can be used to determine PCN insertion as the preferred option over RUS for urinary drainage.

\section{Introduction}

Intrinsic calculus obstructive uropathy is one of the most commonly encountered conditions in daily urological practice. In this situation, urgent temporary drainage of the urinary tract is indispensable, especially in the presence of infection also with infection or higher serum creatinine[1]. In this situation, retrograde ureteral stenting (RUS), percutaneous nephrostomy (PCN) placement, or percutaneous placement of a JJ stent are considered viable options for urinary system drainage [2-4].

\section{Key Words}

Retrograde stenting, ureteral wall density, percutaneous nephrostomy, pyelonephritis, ureteral wall thickness, obstructive uropathy, stone, JJ stent

\section{Competing Interests}

None declared.

\section{Article Information}

Received on February 10, 2021

Accepted on May 1, 2021

Soc Int Urol J.2021;2(4):229-238

DOI: 10.48083/0ZUL6913 


\section{Abbreviations \\ PCN percutaneous nephrostomy \\ P-CUT pericalcular ureteric thickness \\ PUD periureteral density \\ RUS retrograde ureteral stenting \\ URS ureteroscopy}

PCN insertion and RUS have been shown to achieve comparable success rates in patients with obstructive urolithiasis. Nonetheless, rates of sepsis, hospital stay, radiation exposure, and increased costs were higher in patients who were managed by PCN insertion $[5,6]$. Likewise, failure of the retrograde approach for the urinary system might increase the risk of ureteral bleeding, perforation, and stricture formation. There is little evidence to suggest that either RUS or PCN is a better choice as a primary treatment in complicated acute calculus obstruction[7].

Both radiologists and urologists have indicated that the choice of drainage method is largely influenced by personal assessment and stone size[8-10]. In most studies, the larger the stone size, the more likely it was that PCN was chosen as a drainage method. Other factors, including institutional burden and clinician familiarity with the procedure can direct treatment, and there are no universally applied rules for making the choice[11]. In a previous study, the presence of extrinsic obstruction and a higher degree of hydronephrosis were associated with increased risk for RUS failure. Inclusion of heterogeneous intrinsic causes of obstruction in addition to extrinsic causes was a limitation of this study[12].

We aimed to define a precise objective model to select the optimal way to decompress the urinary system obstruction by urolithiasis exclusively. The primary outcome was to define the predictors for initial RUS trial failure in acute complicated calculus obstructive uropathy. The secondary outcome was to delineate the short- and long-term clinical outcomes post initial drainage.

\section{Materials and Methods}

\section{Subjects}

After institutional review board approval, we undertook a retrospective evaluation of patients who presented with acute calculus obstructive uropathy complicated with acute renal failure or obstructive pyelonephritis in a single center between January 2016 and January 2020. Patients were excluded if there was failure to visualize ipsilateral ureteric orifice at the time of RUS or if they had multiple obstructing stones, renal infection (eg, emphysematous pyelonephritis), previous history of urinary diversion or renal transplants, extrinsic ureteral obstruction, or causes of obstruction other than urolithiasis.

Patient demographics and laboratory investigations were collected, including age, gender, body mass index, clinical presentation, medical and surgical history, previous history of ipsilateral stone passage, definitive

\section{FIGURE 1.}

Radiological measurements for P-CUT and PUD at and below the stone level

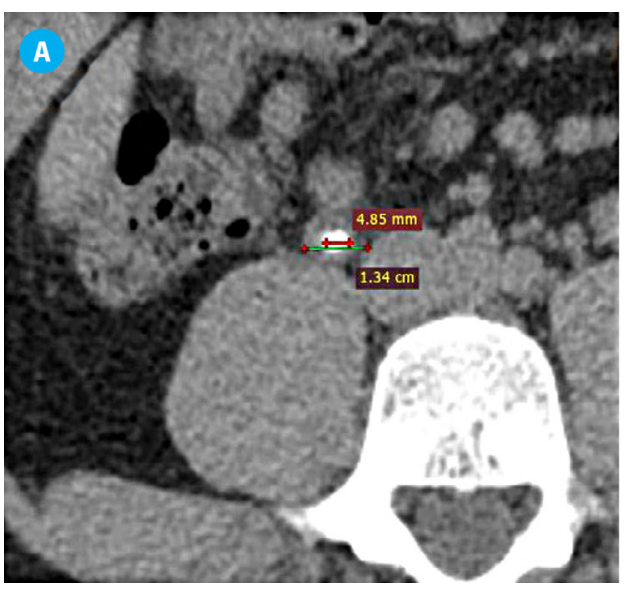

An impacted lumbar ureteral calculus is demonstrated in axial section. The maximum transverse stone diameter $(4.85 \mathrm{~mm})$ is subtracted from the overall ureteral width $(13.4 \mathrm{~mm})$ at this same level to get the P-CUT $(8.55 \mathrm{~mm})$.

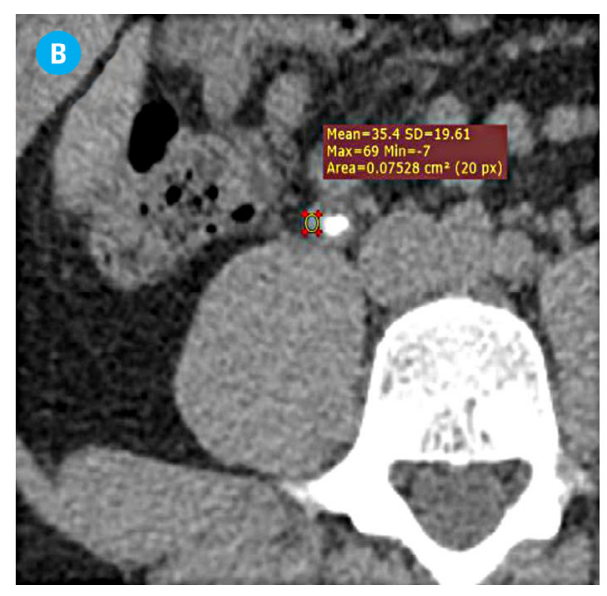

An elliptical region of interest is drawn over the ureter at the level of the calculus (PUD at the level of maximum transverse stone diameter) is $35.4 \mathrm{HU}$.

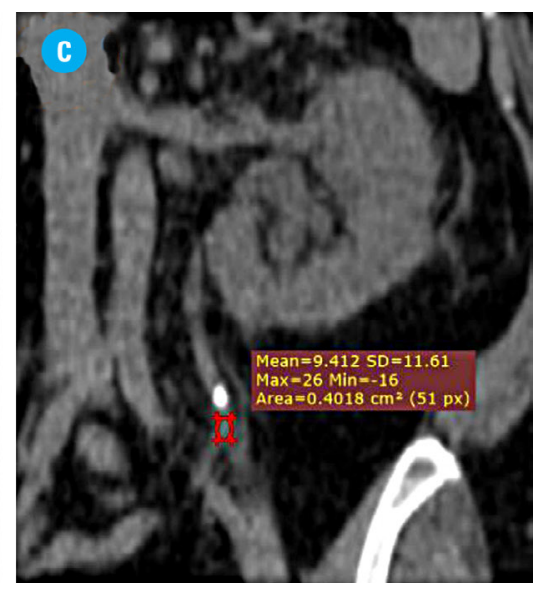

Another region of interest is drawn over the ureter distal to the calculus. (9.4 HU). Average Hounsfield Unit density is calculated by the imaging software. Care is taken not to include the retroperitoneal fat or calculus in the region of interest. 
intervention, and ureteral stenting post definitive intervention, hospital stay, and serum creatinine and leucocytic count at admission.

Radiological parameters were collected using preoperative non-contrast computed tomography $(120 \mathrm{kV}$ and $100 \mathrm{~mA}$, slice thickness was $5 \mathrm{~mm}$ with a $2 \mathrm{~mm}$ overlap using a 64-multislice helical CT scanner [Brilliance, Philips, The Netherlands]), eg, average stone density in Hounsfield unit (HU), laterality, site of the stones (proximal, iliac, or pelvic according to the location to the sacroiliac joints), and degree of hydronephrosis (lower and higher grade) $[13,14]$ were analyzed. Also, maximum transverse stone diameter in $\mathrm{mm}$ was calculated on axial images (Figure 1). Periureteral density (PUD) in HU was measured below the stone level and at the stone level at its maximum transverse diameter[15]. The pericalcular ureteric thickness (P-CUT) was measured as the widest transverse measurement of the ureter involving the stone and the corresponding maximum transverse diameter of the stone at this level. P-CUT was then calculated by subtracting the stone width from the overall stone and ureteral width[16] (Figure 1). Radiological data were interpreted by 2 expert radiologists (> 10 years' experience) blinded to the study objectives.

\section{Methods}

Trial RUS was performed under fluoroscopic guidance by urology senior residents under the supervision of the consultant in charge. With the patient in the lithotomy position, a Terumo hydrophilic guidewire was gently introduced into the collecting system, using a $22 \mathrm{Fr}$ cystoscopy sheath and $30^{\circ}$ telescope, and was followed by the introduction of a 6 Fr open tip catheter (minimal amount of contrast could be injected to ensure the correct position inside the pelvicalyceal system). A 6 Fr JJ stent was later introduced over a PTFE straight guidewire. If the RUS trial failed, patients were referred for ultrasound-guided PCN placement, using Xario 100 system (Toshiba Medical System Corp., Tokyo, Japan) ultrasound scanner, with the patient in the prone position. A 12 or 14 Fr pigtail nephrostomy tube was placed over the guidewire inside the pelvicalyceal system. Contrast was instilled via the PCN to confirm its location in the renal pelvis.

After initial drainage, alpha-blockers were given in case of stones $\leq 10 \mathrm{~mm}$ to promote spontaneous expulsion. Patients were selected for definitive treatment according to European Association of Urology and American Urological Association guidelines[2,3]. A ureteral stent post definitive intervention was inserted

\section{TABLE 1.}

Univariate analysis for RUS failure in acute emergent calcular obstruction

\begin{tabular}{|c|c|c|c|}
\hline Variable & $\begin{array}{c}\text { Failed RUS } \\
(n=48)\end{array}$ & $\begin{array}{l}\text { Successful RUS } \\
\qquad(\mathrm{n}=208)\end{array}$ & $\boldsymbol{P}$-value \\
\hline Age in years (mean $\pm S D$ )* & $60.3 \pm 15.6$ & $57.3 \pm 15.2$ & 0.2 \\
\hline $\begin{array}{l}\text { Gender, n }(\%)^{* *} \\
\text { - Male } \\
\text { - Female }\end{array}$ & $\begin{array}{l}27(56.3) \\
21(43.7)\end{array}$ & $\begin{array}{c}122(58.7) \\
86(41.3)\end{array}$ & 0.4 \\
\hline $\begin{array}{l}\text { Presenting symptoms, } \mathbf{n}(\%)^{* *} \\
\text { - Obstructive pyelonephritis } \\
\text { - Acute renal failure }\end{array}$ & $\begin{array}{l}33(68.8) \\
15(31.2)\end{array}$ & $\begin{array}{c}72(34.6) \\
136(65.4)\end{array}$ & 0.005 \\
\hline $\begin{array}{l}\text { Duration of symptoms before initial drainage in days }{ }^{* * *} \\
\text { (median \& range) }\end{array}$ & $7(3-30)$ & $5(1-21)$ & 0.1 \\
\hline $\begin{array}{l}\text { Medical history, n (\%)** } \\
\text { - Irrelevant } \\
\text { - Diabetes mellitus } \\
\text { - Hypertension }\end{array}$ & $\begin{array}{l}17(35.4) \\
13(27.1) \\
18(37.5)\end{array}$ & $\begin{array}{l}32(15.4) \\
82(39.4) \\
94(45.2)\end{array}$ & 0.2 \\
\hline
\end{tabular}

${ }^{*}$ Independent sample t-test ${ }^{*}$ chi-square test ${ }^{* * *}$ Mann-Whitney test 


\section{TABLE 1.}

Univariate analysis for RUS failure in acute emergent calcular obstruction, Cont'd

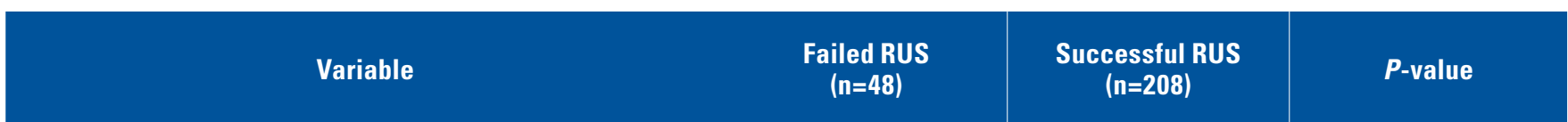

ASA score, $n(\%)^{* *}$

- I

- $\|$

- III

Hx of previous stone passage, $n(\%)^{* *}$

- Yes

- No

Past surgical history on the same side, $\mathrm{n}(\%)^{* *}$

- Irrelevant

- PNL

- SWL

- URS

- Previous JJ stenting

$$
\text { BMI }(\mathrm{Kg} / \mathrm{m} 2)(\text { mean } \pm \mathrm{SD}) \text { * }
$$

Serum creatinine $\mathrm{mg} / \mathrm{dl}$ (median \& range ) ) $^{* * *}$

WBCS at admission $\times 10^{3}(\text { mean } \pm S D)^{*}$

Side, $\mathbf{n}(\%) * *$

- Right

- Left

Stone location, $\mathbf{n}(\%) * *$

- Proximal

- Mid-ureter

- Distal

Degree of hydronephrosis, $\mathbf{n}(\%)^{* *}$

- Low grade

- High grade

Maximum transverse stone diameter in $\mathrm{mm}(\text { mean } \pm \mathrm{SD})^{*}$

Average stone $\mathrm{HU}$ (median \& range) ${ }^{* * *}$

P-CUT at maximum transverse stone diameter in $\mathrm{mm}$ $(\text { mean } \pm S D)^{*}$

PUD at maximum transverse stone diameter in $\mathrm{HU}$ $(\text { mean } \pm S D)^{*}$

PUD below the stone level in HU $(\text { mean } \pm S D)^{*}$
$28(58.3)$

$20(41.7)$

0

17 (35.4)

$128(61.5)$

$31(64.6)$

$80(38.5)$

$110(52.9)$

96 (46.2)

0.4

$2(0.9)$

$(30.5)$

34 (70.8)

$132(63.5)$

$5(10.4)$

$1(2.1)$

$5(10.4)$

$3(6.3)$

$27.4 \pm 6.7$

$1.8(1-13.1)$

$17.2 \pm 6.5$

$25(52.1)$

118 (56.7)

0.4

$23(47.9)$

90 (43.3)

\begin{tabular}{l|l}
\hline $30(62.5)$ & $118(56.7)$ \\
\hline $11(22.9)$ & $40(19.2)$ \\
\hline $7(14.6)$ & $50(24.1)$
\end{tabular}

$30(62.5)$

0.3

50 (24.1)

\begin{tabular}{|r|r|}
\hline $17(35.4)$ & $158(76)$ \\
\hline $31(64.6)$ & $50(24)$ \\
\hline $10.6 \pm 3.5$ & $7.9 \pm 2.7$ \\
\hline
\end{tabular}

$<0.001$

915 (580-1461)

$825(162-1517)$

$<0.001$

$13.5 \pm 6$

$5.5 \pm 2.6$

$<0.001$

$26 \pm 7.9$

$14.2 \pm 6.9$

$<0.001$

$21.6 \pm 10.3$

$11.3 \pm 5.9$

$<0.001$

${ }^{*}$ Independent sample t-test ${ }^{* *}$ chi-square test ${ }^{* * *}$ Mann-Whitney test 
TABLE 2.

Multivariate analysis for predictors of RUS failure

\begin{tabular}{l|l|l|}
\multicolumn{1}{c|}{ Variable } & \multicolumn{2}{|c}{ Multivariable logistic regression analysis } \\
\cline { 2 - 3 } & OR (95\% CI) & P-value \\
\hline Presentation (obstructive pyelonephritis ) & $2.9(1.2-7.2)$ & 0.007 \\
\hline Degree of hydronephrosis (high grade) & $1.1(0.3-6.1)$ \\
\hline Maximum transverse stone diameter $\geq 9.5 \mathrm{~mm}$ & $2.1(1.6-6.8)$ \\
\hline P-CUT at the maximum transverse stone diameter $\geq 7.5 \mathrm{~mm}$ & & 0.8 \\
\hline PUD at the maximum transverse stone diameter $\geq 17.5 \mathrm{HU}$ & $6.1(2.4-15.6)$ \\
\hline PUD below the stone $\geq 19.5 \mathrm{HU}$ & $12.8(4.7-23.1)$ \\
\hline
\end{tabular}

in case of ureteral edema or presence of stone fragments, or to promote healing in case of ureteral injury[17].

\section{Analysis}

Patient demographics and laboratory and radiological data were compared using the chi-square test, independent sample t-test, or Mann-Whitney test as appropriate. Univariate and multivariate analyses were performed to identify factors predicting RUS failure. Receiver operating characteristic (ROC) was used to identify the cut-off value for the best sensitivity and specificity for significant continuous variables in the univariate analysis. All statistical analyses were performed using SPSS version 21 , and $P<0.05$ was considered statistically significant.

\section{Results}

The study included 256 patients who presented with acute complicated calculus obstructive uropathy and were managed initially by RUS trial (Figure 2). Four patients were excluded because of failure to visualize ipsilateral ureteric orifice associated with obstructing stones located in sites in the ureter other than the pelvic ureter ( 2 in the lumbar ureter and others in the iliac ureter away from intramural ureter). Forty-eight patients (18.8 \%) had RUS failure and then were managed by PCN insertion. The patient characteristics were illustrated in Table 1.

In univariate analysis, acute pyelonephritis, higher maximum transverse stone diameter, the higher degree of hydronephrosis, increased P-CUT, and increased PUD below and at the stone level were predictors for initial RUS failure (Table 1). ROC curve was then calculated to identify the cut-off values associated with the best greatest sensitivity and specificity for significant continuous variables in univariate analysis (Figure 3).

In multivariate analysis, acute pyelonephritis, increased the maximum transverse stone diameter $\geq 9.5 \mathrm{~mm}, \mathrm{P}-\mathrm{CUT} \geq 7.5 \mathrm{~mm}$, and PUD at stone level $\geq 17.5$

\section{FIGURE 2.}

Flow chart of the study

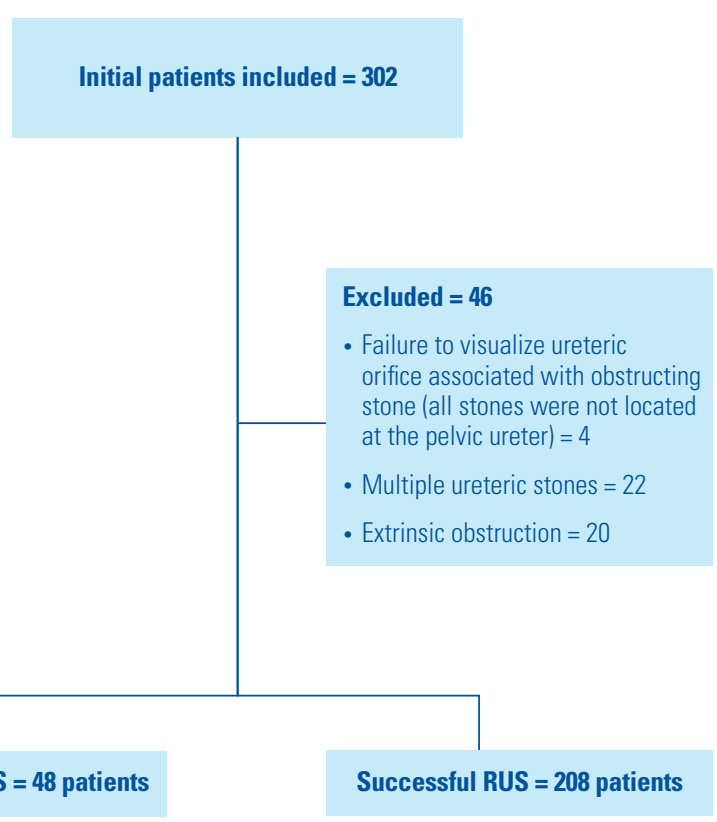




\section{FIGURE 3.}

ROC curve for RUS failure predictors
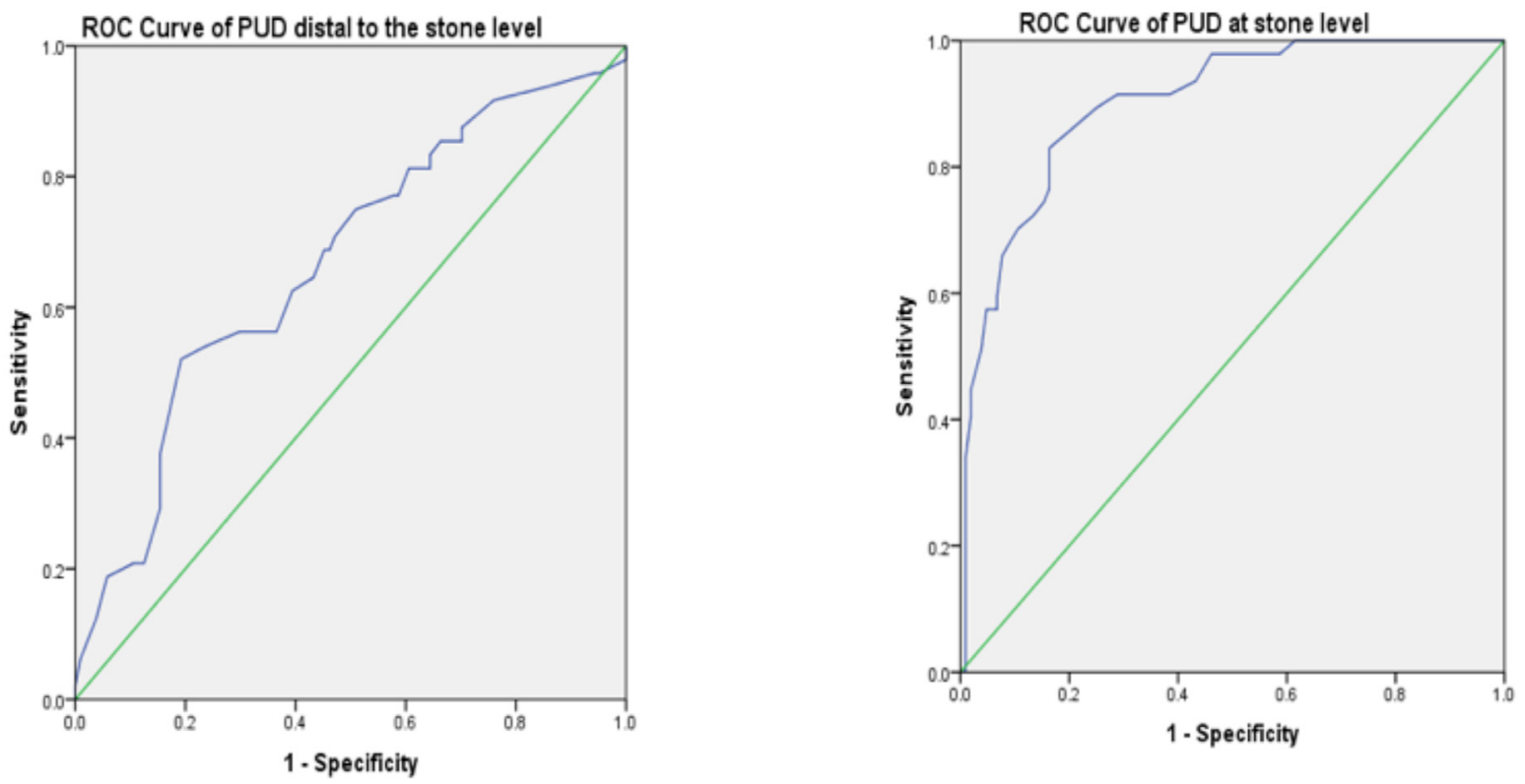

AUC: 0.67

$P$-value: $<0.0001$

Cut-off value: $19.5 \mathrm{HU}$ (sensitivity $67 \%$ and specificity $73 \%$ )

AUC: 0.9

$\boldsymbol{P}$-value: $<0.0001$

Cut-off value: $17.5 \mathrm{HU}$ (sensitivity $83 \%$ and specificity $85 \%$ )

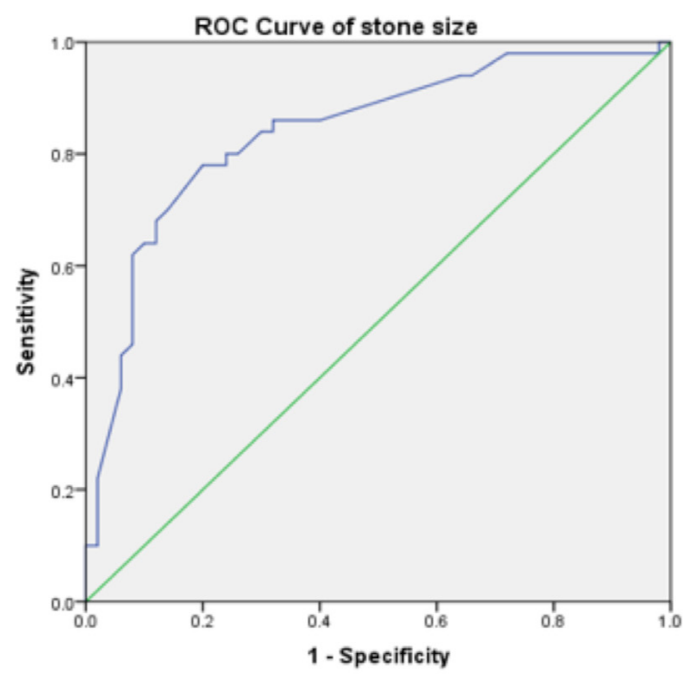

AUC: 0.82

$P$-value: $<0.0001$

Cut-off value: $9.5 \mathrm{~mm}$ (sensitivity $80 \%$ and specificity $75 \%$ )

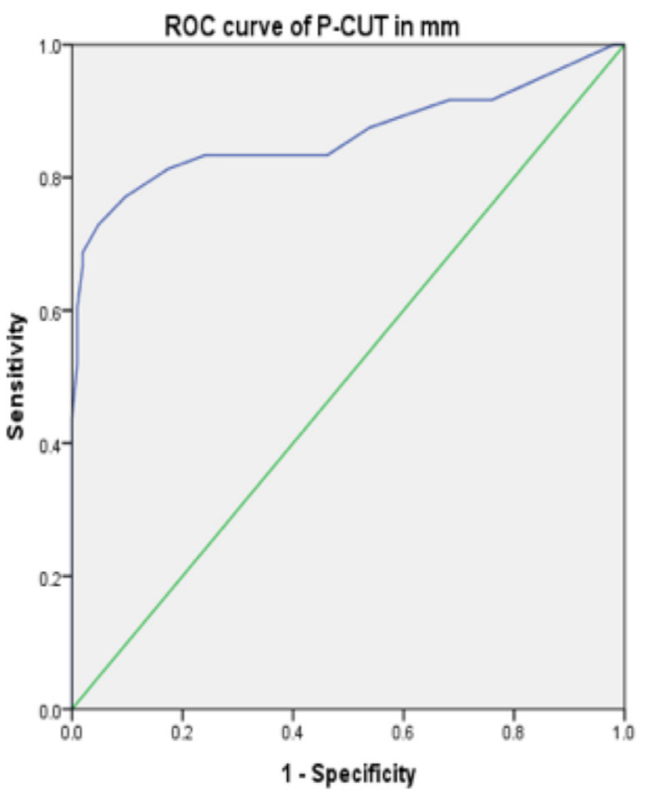

AUC: 0.86

$P$-value: $<0.0001$

Cut-off value: $7.5 \mathrm{~mm}$ (sensitivity $81.5 \%$ and specificity $91 \%)$ 
TABLE 3.

\section{Clinical outcome post RUS trial}

\begin{tabular}{|c|c|c|}
\hline Variable & $\begin{array}{l}\text { RUS failure } \\
\quad(n=48)\end{array}$ & $\begin{array}{c}\text { RUS Success } \\
(n=208)\end{array}$ \\
\hline \multicolumn{3}{|l|}{ Type of management post RUS trial, n (\%) } \\
\hline - Ureteroscopy (semirigid or flexible ) & $24(50)$ & $169(82.6)$ \\
\hline - Percutaneous nephrolithotomy (PNL) & $14(29.2)$ & $20(9.6)$ \\
\hline - Shockwave lithotripsy (SWL) & $7(14.6)$ & $11(5.3)$ \\
\hline - Open surgery & $3(6.3)$ & 0 \\
\hline Duration between initial management and definitive treatment in days & $30(15-120)$ & $20(7-90)$ \\
\hline \multicolumn{3}{|l|}{ Stone status at radiological follow-up, n (\%) } \\
\hline - Static in previous place & $48(100)$ & $135(64.9)$ \\
\hline - Pushed upwards & 0 & $58(27.9)$ \\
\hline - Migrated downwards & 0 & $7(3.4)$ \\
\hline - Passed spontaneously & 0 & $8(3.8)$ \\
\hline Need for JJ stenting post definitive intervention, $n$ (\%) & $23(60.5) / 38$ & $58(30.7) / 189$ \\
\hline - Passage of fragments (without evidence of edema and perforation) & $3(13)$ & $23(39.7)$ \\
\hline - Mucosal laceration and stone impaction & $5(21.7)$ & $1(1.7)$ \\
\hline - Stone impaction without laceration & $15(65.3)$ & $34(58.6)$ \\
\hline
\end{tabular}

HU were associated with increased the risk for RUS failure $(P=0.007,0.002,<0.001$, and $<0.001$, respectively) (Table 2).

The median (range) of the hospital stay between both groups was comparable at the time of temporary drainage $(P=0.4)$ (2[2 to 7] days in RUS failure versus 2 [2 to 10] in RUS success). Only one patient in each group required intensive care unit (ICU) admission due to urosepsis post the primary drainage, and both were subsequently discharged.

In the RUS failure group, ureteral stenting post definitive endourological procedures were required in 23 patients $(60.5 \%)$, and of these, 20 patients (52.6\%) had prior RUS failure due to stone impaction and ureteral mucosal laceration. Of patients with successful RUS, $8(3.8 \%)$ passed the stone spontaneously (mean stone size was $5 \pm 1.2 \mathrm{~mm})$. Ureteral stenting post definitive intervention was required in only 35 patients $(18.5 \%)$ because of stone impaction with a significant difference compared to patients with RUS failure $(P<0.0001)$ (Table 3).

At a median of 14 (6 to 32) months at follow-up, 3 patients were admitted in the RUS failure group. One patient with recurrent stone on the same side and managed by semirigid URS with evidence of passable narrowing below the stone level. The other 2 patients were diagnosed with ureteric strictures; one was managed by open surgery and the other was managed by retrograde laser endoureterotomy).

\section{Discussion}

Stone disease concomitant with pyelonephritis or acute renal failure is a commonly encountered complication in urology. Using RUS, PCN insertion, or percutaneous placement of a JJ stent as temporary relief of the urinary tract obstruction is unavoidable[4,5]. In some situations, using the RUS approach may be associated with intraoperative complications such as the failure of the procedure, retroperitoneal extravasation, abscess formation, and sepsis. Using the PCN as a primary drainage method could guard against these complications.

Opinions on the management of obstructive uropathy vary considerably. Urologists are prone to insert ureteral stents especially in patients with benign disease and in those with coagulopathy $[11,18]$. Studies have indicated that RUS is an efficient method for drainage in $80 \%$ to $100 \%$ of patients in comparison with $95 \%$ to $100 \%$ 
of patients managed by PCN placement in cases with urinary obstruction $[6,12,19]$.

The preoperative physician preference was the main factor controlling the selection of diversion type in patients with obstructive uropathy. Only stone size, independent of other factors, is a reliable predictor of procedure chosen. The overall RUS failure rate in obstructive calculus uropathy complicated with sepsis was $2.3 \%[9]$. In this study, patients who were selected for PCN insertion had a higher stone burden than those selected for RUS (10 [2 to 145] $\mathrm{mm}^{2}$ versus 7[2 to $40] \mathrm{mm}^{2}$ ). According to these data, we could elucidate the lower failure rate during the RUS trial. In another study, the mean stone size was $9.7 \mathrm{~mm}$ in patients who were selected for PCN placement versus $2.6 \mathrm{~mm}$ in patients managed by RUS. Additionally, the median surface area of the stone in patients selected for PCN placement was $92 \mathrm{~mm}^{2}$ versus $47 \mathrm{~mm}^{2}$ in patients managed by RUS $[8,10]$.

The cut-off stone size that would indicate a high risk of RUS failure has yet to be determined. In our study, a maximum transverse stone diameter of $9.5 \mathrm{~mm}$ was associated with 2.1 times increased risk for RUS failure. The RUS failure rate in our study was identified as $18.8 \%$. In addition to the larger stone size in the RUS failure group, we also noticed that the time between the initial symptoms and the first drainage was greater in patients with failed RUS, reflecting greater ureteral inflammation.

Not only stone parameters but also ureteral characteristics on imaging should be considered in the decision to proceed with URS versus PCN. We have identified stone impaction as an additional parameter that predicts clinical outcome $[15,16,20]$. Stone impaction can be explained by pathological changes in the ureteral wall, such as edema and hypertrophy at the site of the stone[21]. This pathological change may in turn lead to endourological procedures failure[22]. In a retrospective study of patients who had undergone ureteroscopic treatment of an impacted ureteral stone, Tran et al. used a Likert scale to classify stone impaction into nonimpacted (1: mobile stone with no edema or 5: stone stuck to the ureter with mild edema) and impacted stones (6: stone stuck in ureter with moderate edema and requiring moderate pressure/irrigation) or 10: stone embedded within ureteral tissue with disimpaction failure.

Numerous studies have highlighted the predictors for stone impaction during URS and determined preoperative radiological parameters such as increased ureteral wall thickness and ureteral wall density at the stone level to be predictors for stone impaction $[15,16,23]$. Higher PUD below the stone level has been considered to be another co-factor responsible for URS failure as it may represent more ureteral inflammation and spasm precluding passage of the ureteroscope to reach stones $[15,16]$. Other studies have found the severity of hydronephrosis and increased serum creatinine to be associated with impacted ureteral stones during URS $[24,25]$. In our study, neither PUD below the stone level nor the degree of hydronephrosis was a significant predictor for RUS failure. Conversely, P-CUT $\geq 7.5$ $\mathrm{mm}$ and PUD at maximum transverse stone diameter $\geq 17.5 \mathrm{HU}$ were associated with a 6.1-fold and a 12.8fold increase, respectively, in the rate of RUS failure necessitating subsequent PCN insertion for emergent urinary drainage.

Pearl et al. showed that RUS failure was more obvious in the elderly with high grade fever[19]. Also, in previous studies predicting RUS failure in the presence of intrinsic obstructive factors rather than urolithiasis, including male sex, a higher degree of hydronephrosis, and increased serum creatinine at presentation $[12,26]$. Acute pyelonephritis was a predictor of RUS failure in our experience. This could be indicated by the presence of a higher degree of hydronephrosis, urine stasis, and pathogen proliferation for a prolonged period before sepsis occurrence.

Spontaneous stone passage and downward displacement of stones were encountered in $3.8 \%$ and $3.4 \%$ of patients with successful URS, respectively. Yoshida et al. concluded that in addition to the passive ureteral dilation caused by the ureteral stent, ureteral wall thickness is a substantial factor for spontaneous passage of ureteric stones $\leq 10 \mathrm{~mm}[27]$. On the contrary, it was reported that spontaneous stone passage in patients with PCN was higher than in patients with RUS[8]. In our study, all patients in the RUS failure group had a static stone position after the initial drainage and before the definitive intervention. Higher PUD below the stone level and at the stone level in addition to increased P-CUT might explain the progressive ureteral inflammation and spasm that hindered stone movement.

Cevik et al. showed that routine placement of a ureteral stent is not mandatory in patients without complications after URS for impacted ureteral stones[17]. Even though the time interval between emergent urinary drainage and definitive endurological stone treatment was short, the rate of ureteral stenting in patients with successful RUS was only $18.5 \%$. The rate of stenting was higher at $52.6 \%$ in patients with initial failed RUS. There was a higher rate of ureteral stenting in patients with RUS failure after the definitive intervention: $52.6 \%$ versus $18.5 \%$ in patients with successful RUS. Also, the rates of ureteral laceration associated with stone impaction during definitive intervention and ureteral stricture occurrence during follow-up were higher in patients with RUS failure. Morgentaler et al. 
higher in patients with RUS failure. Morgentaler et al. concluded that $28.5 \%$ of patients (12/42) with impacted ureteral stones developed ureteral trauma during stone manipulation. Two patients later developed ureteral stricture[28]. In addition to the beneficial role of alphablockers use to facilitate ureteral dilation and stone access during ureteroscopy[29,30]. The use of NSAIDS and/or chymotrypsin as add-on therapy in severely impacted stones before definitive intervention has also been suggested.

The study was limited by small sample size and inherent selection bias due to the retrospective nature of the study. Failure to assess the quality of life, lack of radiation exposure estimation, and financial cost calculation were other limitations.

\section{References}

1. Campbell S, Lane B. Malignant renal tumors In: Wein AJ, Kavoussi LR, Partin AW, Peters CA., eds. Campbell-Walsh Urology. Philadelphia, PA, USA: Elsevier; 2016.

2. Assimos D, Krambeck A, Miller NL, Monga M, Murad MH, Nelson CP, et al. Surgical management of stones: American urological association/endourological society guideline, PART I. J Urol.2016;196(4):1153-1160.

3. Turk CNA, Petrik A, Seitz C, et al. (2020) Guidelines on urolithiasis. https://uroweb.org/guideline/urolithiasis/.

4. Masood J, Ismail M, El-Husseiny T, Moraitis K, Albanis S, Papatsoris $A$, et al. 'An interventional urology list'-a novel concept for UK urological services. Ann R Coll Surg Engl.2011;93(1):27-30.

5. Weltings S, Schout BM, Roshani H, Kamphuis GM, Pelger RC Lessons from Literature: Nephrostomy Versus Double J Ureteral Catheterization in Patients with Obstructive Urolithiasis-Which Method Is Superior? J Endourol.2019;33(10):777-786.

6. Skolarikos A, Alivizatos G, Papatsoris A, Constantinides K, Zerbas A, Deliveliotis C. Ultrasound-guided percutaneous nephrostomy performed by urologists: 10-year experience. Urology.2006;68(3):495-499.

7. Türk C, Petřík A, Sarica K, Seitz C, Skolarikos A, Straub M, et al. EAU guidelines on interventional treatment for urolithiasis. Eur Urol.2016;69(3):475-482.

8. de Sousa Morais N, Pereira JP, Mota P, Carvalho-Dias E, Torres JN, Lima E. Percutaneous nephrostomy vs ureteral stent for hydronephrosis secondary to ureteric calculi: impact on spontaneous stone passage and health-related quality of life-a prospective study. Urolithiasis.2019:47(6):567-573.

9. Goldsmith ZG, Oredein-McCoy 0, Gerber L, Bañez LL, Sopko DR, Miller MJ, et al. Emergent ureteric stent vs percutaneous nephrostomy for obstructive urolithiasis with sepsis: patterns of use and outcomes from a 15-year experience. BJU Int.2013;112(2):E122-E8.

\section{Conclusion}

In acute obstructive uropathy complicated with acute pyelonephritis and associated with a stone diameter around $1 \mathrm{~cm}$, it is not only the stone profile index that is responsible for RUS failure. The ureteral profile is also substantial finding which can help in drainage type selection. Increased P-CUT $\geq 7.5 \mathrm{~mm}$, and PUD $\geq 17.5 \mathrm{HU}$ were predictive parameters for initial RUS trial failure and the necessity of PCN placement. In this group of patients after definitive intervention, ureteral stenting is still needed in $52.6 \%$ due to persistent ureteral edema and lacerations. Pre-definitive intervention use of alpha-blockers besides NSAIDS or chymotrypsin may reduce stone impaction ureteral lacerations and facilitate stone passage.

10. Yoshimura K, Utsunomiya N, Ichioka K, Ueda N, Matsui Y, Terai A. Emergency drainage for urosepsis associated with upper urinary tract calculi. J Urol.2005;173(2):458-462.

11. Lynch MF, Anson KM, Patel U. Current opinion amongst radiologists and urologists in the UK on percutaneous nephrostomy and ureteric stent insertion for acute renal unobstruction: Results of a postal survey. BJU Int.2006;98(6):1143-1144.

12. Yossepowitch O, Lifshitz DA, Dekel Y, Gross M, Keidar DM, Neuman $M$, et al. Predicting the success of retrograde stenting for managing ureteral obstruction. J Urol.2001;166(5):1746-1749.

13. Elbaset M, Ezzat O, Elgamal M, Sharaf M, Elmeniar A, Abdelhamid $A$, et al. Supranormal differential renal function in adults with ureteropelvic junction obstruction: does it really exist? Indian J Urol.2020;36(3):205.

14. Elbaset MA, Osman Y, Elgamal M, Sharaf MA, Ezzat O, Elmeniar $A M$, et al. Long-term outcomes after pyeloplasty for pelvi-ureteric junction obstruction in adults associated with renal congenital anomalies: age, sex and renal function matched analysis. Arab $\mathrm{J}$ Urol.2020:1-6.

15. Tran TY, Bamberger JN, Blum KA, Parkhomenko E, Thai J, Chandhoke RA, et al. Predicting the impacted ureteral stone with computed tomography. Urology.2019;130:43-47.

16. Chandhoke R, Bamberger JN, Gallante B, Atallah W, Gupta M. Peri-calculus ureteral thickness on computed tomography predicts stone impaction at time of surgery: a prospective study. $J$ Endourol.2020;34(1):107-111.

17. Cevik I, Dillioglugil O, Akdas A, Siegel Y. Is stent placement necessary after uncomplicated ureteroscopy for removal of impacted ureteral stones? J Endourol.2010;24(8):1263-1267.

18. Sammon JD, Ghani KR, Karakiewicz PI, Bhojani N, Ravi P, Sun M, et al. Temporal trends, practice patterns, and treatment outcomes for infected upper urinary tract stones in the United States. Eur Urol.2013;64(1):85-92. 
19. Pearle MS, Pierce HL, Miller GL, Summa JA, Mutz JM, Petty BA, et al. Optimal method of urgent decompression of the collecting system for obstruction and infection due to ureteral calculi. J Urol.1998;160(4):1260-1264.

20. Elbaset M, Elkarta A, Eraky A, Badawy M, Sheir K, Shokeir A. Role of pretreatment Doppler ultrasound in the prediction of factors affecting stone-clearance post-shockwave lithotripsy for ureteral stones: a prospective study. Int Urol Nephrol.2020;52(9):1643-1649. doi: 10.1007/s11255-020-02465-3. Epub 2020 Apr 15.

21. Brito AH, Mitre Al, Srougi M. Ureteroscopic pneumatic lithotripsy of impacted ureteral calculi. Int Braz J Urol.2006;32(3):295-299.

22. Oliver R, Wells H, Traxer 0, Knoll T, Aboumarzouk 0, Biyani CS, et al. Ureteric stents on extraction strings: a systematic review of literature. Urolithiasis.2018;46(2):129-136.

23. Legemate JD, Wijnstok NJ, Matsuda T, Strijbos W, Erdogru T, Roth $B$, et al. Characteristics and outcomes of ureteroscopic treatment in 2650 patients with impacted ureteral stones. World J Urol.2017;35(10):1497-1506.

24. Tran TY, Hernandez Bustos N, Kambadakone A, Eisner B, Pareek G. Emergency ureteral stone treatment score predicts outcomes of ureteroscopic intervention in acute obstructive uropathy secondary to urolithiasis. J Endourol.2017;31(9):829-834.
25. Sarica K, Eryildirim B, Sahin C, Sabuncu K, Cetinel C, Narter F. Impaction of ureteral stones into the ureteral wall: Is it possible to predict? Urolithiasis.2016;44(4):371-376.

26. Wenzler DL, Kim SP, Rosevear HM, Faerber GJ, Roberts WW, Wolf J, J Stuart. Success of ureteral stents for intrinsic ureteral obstruction. J Endourol.2008;22(2):295-300.

27. Yoshida T, Inoue T, Taguchi M, Omura N, Kinoshita H, Matsuda $\mathrm{T}$. Ureteral wall thickness as a significant factor in predicting spontaneous passage of ureteral stones of $\leq 10 \mathrm{~mm}$ : a preliminary report. World J Urol.2019;37(5):913-919.

28. Morgentaler A, Bridge SS, Dretler SP. Management of the impacted ureteral calculus. J Urol.1990;143(2):263-266.

29. Alsaikhan B, Koziarz A, Lee JY, Pace KT. Preoperative alphablockers for ureteroscopy for ureteral stones: a systematic review and meta-analysis of randomized controlled trials. J Endourol.2020;34(1):33-41.

30. Koo KC, Yoon J-H, Park N-C, Lee HS, Ahn HK, Lee KS, et al. The impact of preoperative $\alpha$-adrenergic antagonists on ureteral access sheath insertion force and the upper limit of force required to avoid ureteral mucosal injury: a randomized controlled study. J Urol.2018;199(6):1622-1630. 